\title{
$\beta$-Elemene: Mechanistic Studies on Cancer Cell Interaction and Its Chemosensitization Effect
}

\author{
Ziyu Jiang ${ }^{1,2}$, Joe A. Jacob ${ }^{1}$, Dinesh S. Loganathachetti ${ }^{3}$, Prasannabalaji Nainangu ${ }^{4}$ and \\ Baoan Chen ${ }^{1 *}$ \\ ${ }^{1}$ Department of Hematology and Oncology, Zhongda Hospital, School of Medicine, Southeast University, Nanjing, China, \\ ${ }^{2}$ Department of Oncology, Affiliated Hospital of Integrated Traditional Chinese and Western Medicine, Nanjing University of \\ Chinese Medicine, Nanjing, China, ${ }^{3}$ Department of Marine Biotechnology, Bharathidasan University, Tiruchirappalli, India, \\ ${ }^{4}$ Department of Microbiology, Bharathidasan University, Tiruchirappalli, India
}

Over the past decade, screening and identifying novel compounds for their biomedical applications has become an upcoming area of research. Identifying the molecular mechanisms of these compounds has become an integral part of anticancer research. $\beta$-elemene, a sesquiterpene, is renowned for its anticancer activity against a variety of cell lines. Recent studies on $\beta$-elemene have elucidated that it possesses antiproliferative effect on cancer cells by creating an apoptotic trigger. Interestingly, it also induces protective autophagy in some cancerous cell lines and is less cytotoxic compared to other widely accepted chemotherapeutic agents. This provides an edge with the perception of limited toxicity to normal cells. This mini-review precisely focuses on the studies performed to identify the mechanism of anticancer activity of $\beta$-elemene against cancer cells of multiple origin. In accordance to the evaluation made by the studies mentioned, apoptosis has been identified to be most possible reason behind anticancer activity exerted by $\beta$-elemene against a variety of cancer cell lines. Cell cycle arrest and necrosis have been credited to be possible alternate mechanisms for the anticancer effect of $\beta$-elemene.

Keywords: $\beta$-elemene, anticancer, apoptosis, cytotoxic, mechanism

\section{INTRODUCTION}

Cancer is the reason behind highest number of deaths across the world and contemporary measures have become the need of the hour (Siegel et al., 2016). $\beta$-elemene is a sesquiterpene, with a wide spectrum of antineoplastic activity, even against drug-resistant tumors and other complex malignancies (Liu et al., 2011). Among its three isomers $(\delta, \alpha, \beta), \beta$-elemene (referred to as elemene, hereafter), is the predominant component (Wu et al., 2009).

The most well studied mechanism by which anticancer agents induce cell death in cancer cells is by the induction of apoptosis (Frankfurt and Krishan, 2003). DNA fragmentation and activation of caspases are the major hallmarks of apoptosis (Saraste and Pulkki, 2000). Apoptosis has been strongly induced in cancerous cells by elemene (Guan et al., 2014). Elemene, a prominent component of traditional chinese medicine (TCM) regulates several molecular targets and exerts promising anticancer effects (Jiang et al., 2016). Although elemene can induce antitumor effects in cancerous cells of multiple origin, the mechanism of action still remains unclear (Zhu et al., 2011b). In the current mini-review, the mechanism of action of elemene was reviewed and analyzed. 


\section{ELEMENE MECHANISM OF ACTION AGAINST CANCER CELLS OF MULTIPLE ORIGIN}

\section{Integumentary System}

In B16F10 melanoma cells, the expression of VEGF was inhibited by elemene elucidating its antiangiogenic activity. Similar results were obtained in rat aortic ring and chicken embryo chorioallantoic membrane. In C57BL/6 melanoma mice, western blot and immunohistochemical analysis showed that CD34 expression was reduced, further evidenced by reduction in tumor volume and suppression of VEGF-mediated angiogenesis. This study therefore determines that the antineoplastic effect of elemene in cells of melanoma origin is by an antiangiogenic effect (Chen et al., 2011).

\section{Nervous System}

Mitogen-activated protein kinase kinase-3 (MKK3) and -6 (MKK6), is a kinase enzyme which phosphorylates mitogen-activated protein kinase (MAPK). When activated through mutual compensation, it can mediate the antiglioblastoma effect of elemene. In the human U87 glioblastoma cell line, the upregulation of MKK3 and MKK6 can lead to cell cycle arrest at the G0/G1 phase thereby leading to an anticancer effect of elemene (Zhu et al., 2011b). Elemene, in a similar study, inhibited the proliferation of U87 cells through the Glia maturation factor $\beta$-dependent inactivation of the ERK1/2$\mathrm{Bcl}-2$ /survivin pathway. It also chemosensitized the glioblastoma cells to temozolomide (Zhu et al., 2014). In C6 and U251 glioblastoma cells, elemene treatment resulted in p38 MAPK phosphorylation and cell-cycle arrest at the G0/G1 phase (Yao et al., 2008a,b). Activation of Glia maturation factor $\beta$ mediates the antitumor effect of elemene in glioblastoma cell line U87 and this can increase the sensitivity of cancer cells to cisplatin (Zhu et al., 2011a). Therefore, the antiglioblastoma effect of elemene is mediated through the cell cycle arrest at G0/G1 phase, leading to MAPK phosphorylation. The p38 MAPK phosphorylation is activated by the upregulation of MKK3 and MKK6 (Son et al., 2010). The activation of p38 MAPK eventually leads to cellular apoptosis (Koul et al., 2013). Therefore, these studies are an evidence for killing of glioblastoma cells by elemene via an apoptotic trigger.

Supporting this view, elemene increased caspase-3/7/10 activity, up-regulated Bax expression, and down-regulated the Bcl-2, Bcl-XL, and of X-linked inhibitor of apoptosis expressions. This suggests the role of apoptosis in anticancer effect of elemene on brain tumor cell lines A172, CCF-STTG1, and U-87MG (Li et al., 2013b). Elemene induced cell cycle arrest at G0/G1 phase in U87 cells and elevated the expressions of caspases-3, -8, -9, Fas, FasL, and Bax. It downregulated the expressions of Bcl-2, indicating the induction of apoptosis in the cancer cells (Li et al., 2014). In glioma U251 and A172 cells, elemene induced apoptosis through upregulation of caspase-9, -3 and -7 expressions and downregulation of survivin gene expression (Zhang et al., 2012). Against the rat glioma cell C6 and human glioma cell SHG-44, elemene possessed antiproliferative effects through induction of apoptosis (Zhou et al., 2003). These studies prove that the antineoplastic effect of elemene against cancers of nervous system is by the induction of apoptosis.

\section{Respiratory System}

In the human NSCLC cell lines H460 and A549, a Chk2dependent mechanism adopted by elemene caused the G2$\mathrm{M}$ arrest. Elemene induced caspase-3, -7 , and -9 activities, decreased Bcl-2 expression, resulted in cytochrome c release and increased the levels of cleaved caspase- 9 and poly (ADPribose) polymerase through a mitochondrial release of the cytochrome c-mediated apoptotic pathway (Wang et al., 2005). Elemene and etoposide phosphate in synergism can induce the expressions of Bax, p53, and p21, mediated by cleavage of PARP and the suppression of cyclin D1 in A549 NSCLC cells (Zhang et al., 2011). Elemene can restore the sensitivity of NSCLC cells (PC9 and H1299) to Gefitinib by upregulation of p21 expression (Zhao et al., 2011). p21 is a key arbitrator of p53 function, which can lead to apoptosis (Xia et al., 2011). Elemene can induce the expressions of Bax and phosphoBcl-2 and decrease Bcl-2 and XIAP expressions in human NSCLC cell lines $\mathrm{H} 460$ and A549. It can also increase the cisplatin-induced expressions of caspase-3, -7, -9, and -10 activities and cleaved caspase-3, -9, and poly (ADP-ribose) polymerase levels thereby sensitizing the cells to cisplatin by mitochondria-mediated intrinsic apoptosis pathway (Li et al., 2009). Elemene has antiproliferative effects on human NSCLC PC9, H1299, H1650, A549, H358, and H1975 cells by ERK1/2and $\mathrm{AMPK} \alpha$-mediated inhibition of transcription factor $\mathrm{Sp} 1$, followed by reduction in DNMT1 protein expression (Zhao et al., 2015).

Elemene in synergism with Docetaxel resulted in increased cytochrome $c$ release from mitochondria, significant caspase8 and -3 cleavage, and downregulation of $\mathrm{Bcl}-2$ and $\mathrm{Bcl}-$ XL expressions in p53 mutant $\mathrm{H} 23$ cells and p53 null H358 cells thereby leading to a p53- and Fas-independent pathway via mitochondria (Zhao et al., 2007). In laryngeal HEp-2 cells, in vitro and HEp-2 cell-transplanted BALB/c nude mice, in vivo, elemene enhanced the expression of caspase-3, thereby inhibiting the eukaryotic initiation factors (eIF4E and eIF4G), basic fibroblast growth factor (bFGF), and vascular endothelial growth factor (VEGF) (Tao et al., 2006). Collectively, the results of these studies elucidate that the antineoplastic effect of elemene in cancers of respiratory system is by the induction of apoptosis.

\section{Immune System}

Five piperazine derivatives of $\beta$-elemene were synthesized and their antileukemic effect was tested against HL-60, NB4, K562m and HP100-1. DX-1 was effective in induction of apoptosis in HL-60 cell line by both receptor and mitochondria mediated pathways. Caspase- 8 activation was correlated with the decrease in the levels of cellular FLICE-inhibitory protein (c-FLIP), an inhibitor of apoptosis triggered by engagement of death receptors (Yu et al., 2011). When treated against HL-60 cells, elemene enhanced the apoptotic effect induced by aclarubicin and down-regulated COX-2, NF-kappaB and PGE2 expressions 
(Zheng et al., 2009). When elemene was treated against human multiple myeloma cell line RPMI-8226, caspase-3, and DR4 protein expressions increased, whereas, bcl-2, NF-kappaB and P65 protein expressions decreased. Apoptosis induced by elemene may be by activation of mitochondrial and death receptor mediated pathways, according to the authors (Chen et al., 2010).

An elemene derivative named $\mathrm{N}$-(beta-elemene-13-yl) tryptophan, treated against human acute promyelocytic leukemia NB4 cells resulted in apoptotic cell death mediated by hydrogen peroxide generation and activation of caspase-3 (Yu, 2010). Taken together, elemene exerts its antineoplastic on cancers of immune system through induction of apoptosis.

\section{Digestive System}

The copper transporter 1 levels are increased by treatment with elemene, which can allow the entry of oxaliplatin into hepatocellular carcinoma cell lines $\mathrm{MHCC} 97 \mathrm{H}$ and Hep3B. This will result in increased sensitivity of cancer cells to oxaliplatin (Li et al., 2016). Against the hepatoma cell line HepG2, elemene can induce cell cycle arrest at $\mathrm{G}_{2} / \mathrm{M}$ phase and cell death by apoptosis by upregulating Fas/FasL expression (Dai et al., 2013). Multidrug resistant variant human gastric adenocarcinoma SGC7901/ADM cell line was treated with elemene. Apoptosis was observed with increasing concentrations of elemene and it also increased the sensitivity of the cell line to adriamycin which might be due to inhibition of NF-kappaB activity (Fu et al., 2013). Elemene suppressed the proliferation of esophageal carcinoma ECA-109 cells by inhibiting the human telomerase reverse transcriptase expression using a long noncoding RNA CDKN2B-AS1 (Hu et al., 2015). These results also stand supportive for the antineoplastic effect of elemene through an apoptotic trigger.

\section{Reproductive System}

Elemene inhibited the proliferation of androgen-insensitive prostate carcinoma DU145 and PC-3 cells and induced apoptosis as observed by TUNEL assay and flow cytometric analysis. This was further evidenced by decreased levels of bcl-2, increased levels of cytochrome c release, activated PARP and caspase- 3 , $-7,-9$, and -10 in prostate cancer cells after elemene treatment (Li et al., 2010a). In a similar study, elemene treatment against the androgen-independent prostate carcinoma DU145 and PC-3 cells resulted in mitochondria mediated apoptosis. Elemene acted as a chemosensitizing agent for cisplatin in this study by augmenting the cisplatin-induced activation of caspases-3, 7, 9, and 10, cleavage of caspase-3 and -9, suppression of $\mathrm{Bcl}-2$ and $\mathrm{Bcl}-\mathrm{XL}$ expression, and release of cytochrome $c$ from the mitochondria of these cells (Li et al., 2010b).

In the cisplatin-sensitive human ovarian cancer cell line A2780 and its cisplatin-resistant counterpart A2780/CP, treatment of elemene alone or in synergism with cisplatin, resulted in alterations in cyclin and cyclin-dependent kinase expression, including the down-regulation of $\mathrm{CDC} 2$, cyclin $\mathrm{A}$, and cyclin $\mathrm{B} 1$, and the up-regulation of $\mathrm{p} 21 \mathrm{WAF} 1 / \mathrm{CIP} 1$ and $\mathrm{p} 53$ proteins caused cell cycle arrest at the G2/M phase. There was an apoptotic trigger which was an outcome of the activation of caspases-3, -8 , and -9 , the loss of mitochondrial membrane potential $(\Delta \Psi \mathrm{m})$, the release of cytochrome $\mathrm{c}$ into the cytosol and changes in the expression of bcl-2 family proteins ( $\mathrm{Li}$ et al., 2005; Lee et al., 2012). Similar results were obtained by yet another study, with elemene inducing a potential apoptotic trigger (Lee et al., 2012). In the breast cancer MB-468 cells, elemene in synergism with paclitaxel can down-regulate the cell cycle protein cyclin-B1 expression and up-regulate the P27(kip1) expression thereby leading to an inhibition of cell proliferation (Cai et al., 2013). The human cisplatin-resistant ovarian cancer cell lines A2780/CP and MCAS were chemosensitized by elemene through the induction of apoptosis, mediated by a mitochondria- and caspase-dependent (caspase-3/8/9) cell death pathway. It upregulated the expression of pro-apoptotic Bax and downregulated the anti-apoptotic Bcl-2 and Bcl-XL expressions (Li et al., 2013c).

Therefore, the cancers of reproductive system are susceptible to elemene via an apoptotic trigger as observed through the outcomes of these experiments.

\section{Endocrine System}

Synergistic interaction of rapamycin and elemene influenced a significant antiproliferative effect in FTC-133 thyroid cell line, dependent on inhibiting the AKT feedback activation induced by Rapamycin (Zhou et al., 2016). Inhibition of Akt activity by Rapamycin has been related to induction of apoptosis (Dormond et al., 2007). Therefore, the inhibition of Akt by Rapamycin in this study could be correlated to an apoptotic trigger.

\section{Urinary System}

Elemene enhanced the activity of cisplatin by chemosensitization against human bladder cancer 5637 and T-24 cells by elevating expressions of caspase- $3,-7,-8,-9$, and -10 leading to apoptosis through a caspase dependent mechanism (Li et al., 2013e). Elemene downregulates the expression of survivin, Bcl-xL, Mta1 and induces apoptosis in human bladder cancer T24 cells in a time and dose dependent manner (Chen et al., 2012). This suggests that apoptosis is the rationale behind the observed antineoplastic effects in cancers of urinary system.

\section{SENSITIZATION OF MULTIDRUG RESISTANT CANCER CELLS}

Elemene increased the susceptibility of multidrug resistant cancer cells toward the respective drugs in several cases. It increased the sensitivity of MDR leukemia (K562/DNR) and gastric cancer lines (SGC7901/ADR), thereby enhancing the cytotoxic activity of P-gp substrates (DOX, DNR, and EPI). This was achieved by downregulation of Akt phosphorylation and upregulation of the E3 ubiquitin ligases, c-Cbl and Cbl-b (Zhang et al., 2013). In combination with anti-neoplastic drugs (colchicine, vinblastine and paclitaxel), elemene was reported to produce effective cytotoxicity by blocking efflux portion of ABCB1 transporters that are over-expressed in KB-C2 cells (Guo et al., 2014). In 
TABLE 1 | Anticancer effect of $\beta$-elemene against cancer cell lines of multiple origin.

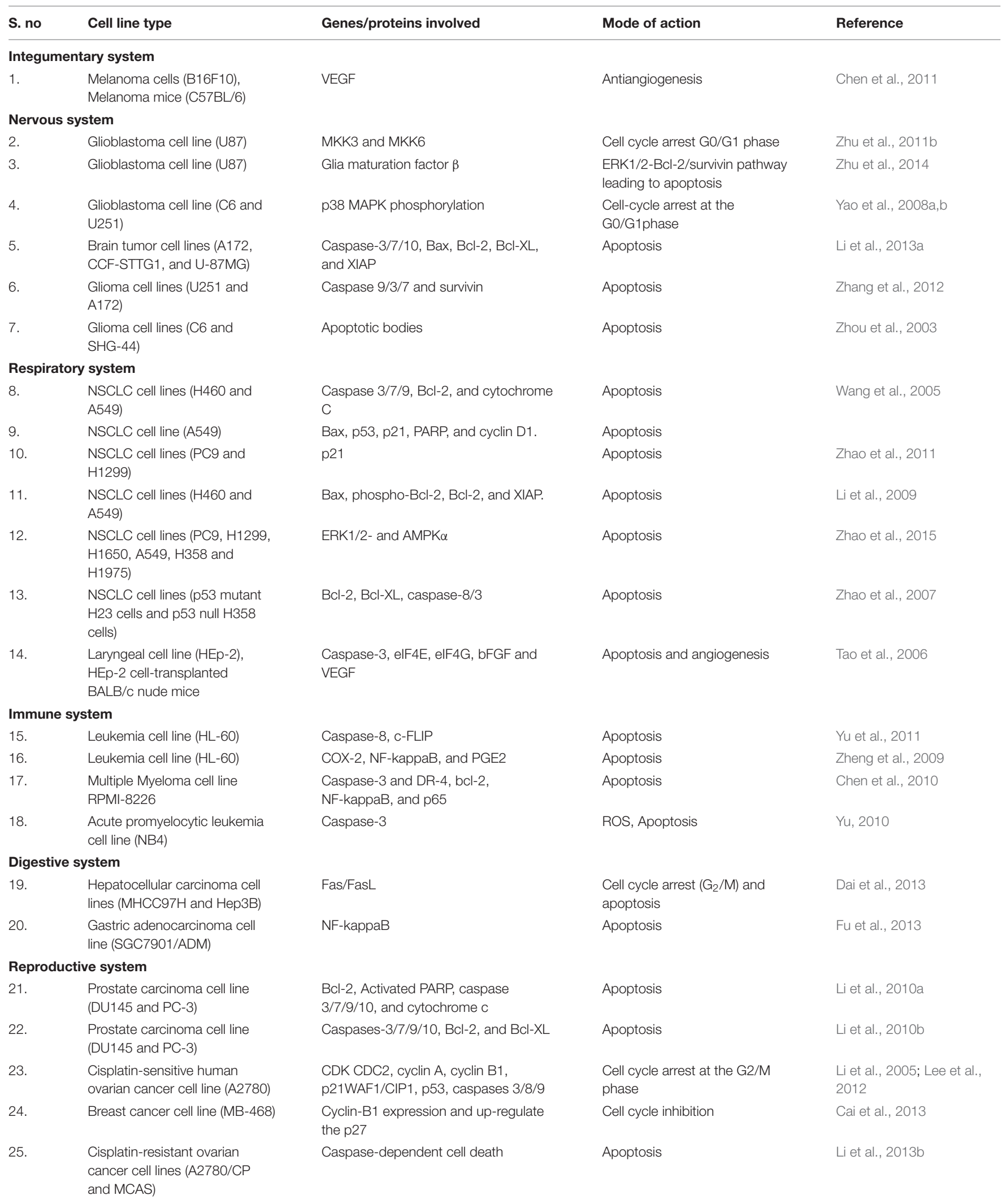


TABLE 1 | Continued

\begin{tabular}{|c|c|c|c|c|}
\hline S. no & Cell line type & Genes/proteins involved & Mode of action & Reference \\
\hline \multicolumn{5}{|c|}{ Endocrine system } \\
\hline 26. & Thyroid cell line (FTC-133) & AKT feedback activation & Apoptosis & Zhou et al., 2016 \\
\hline 27. & Bladder cancer cell line (5637 and T-24) & Caspase 3/7/8/9/10 & Apoptosis & Li et al., 2013c \\
\hline 28. & Bladder cancer cell line (T24) & Bcl-xL, Mta-1, and survivin & Apoptosis & Chen et al., 2012 \\
\hline
\end{tabular}

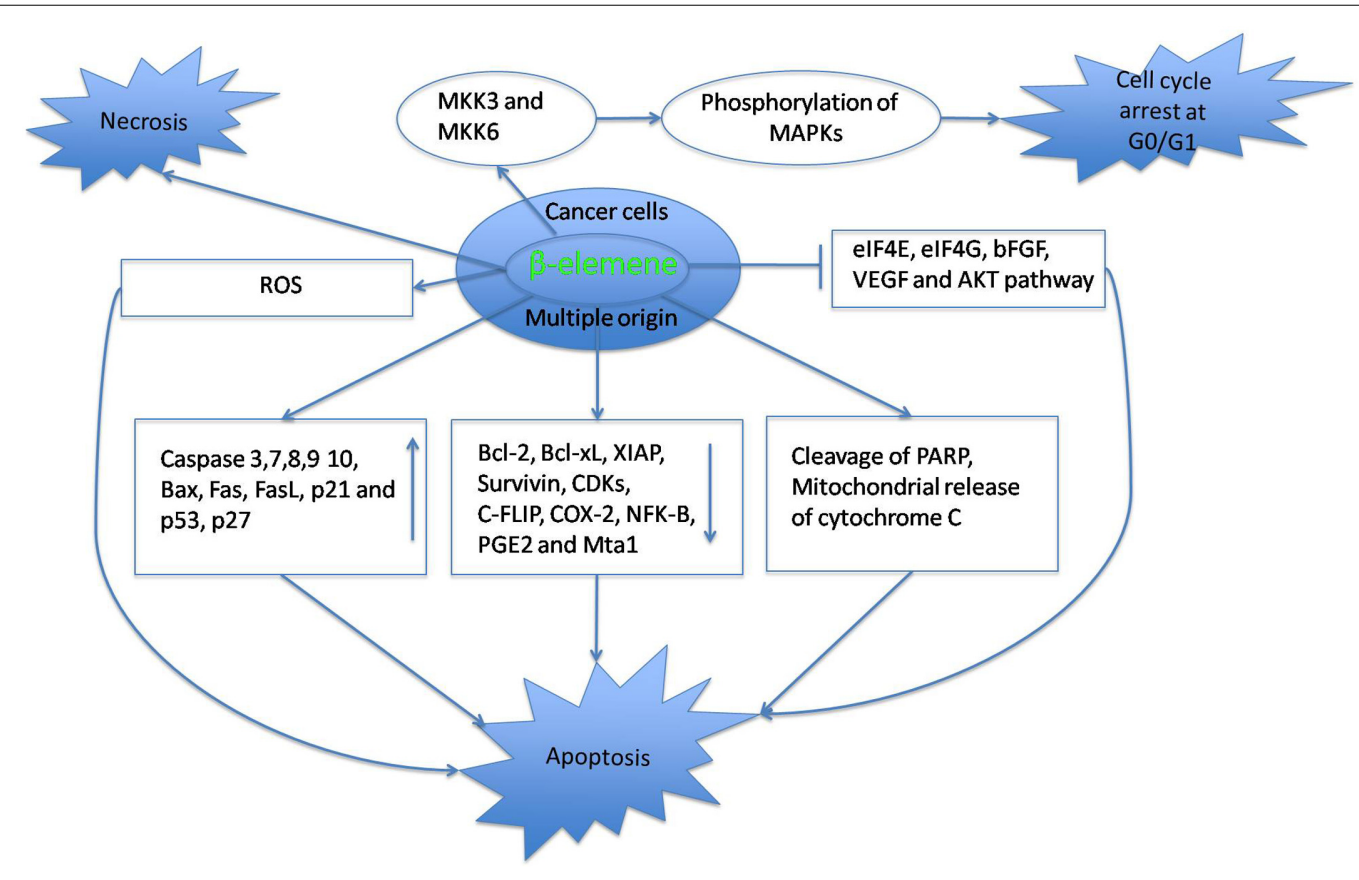

FIGURE 1 | Modes of cell death induced by $\beta$-elemene.

human BCA adriacin (Adr) - resistant MCF-7 cells (MCF-7/Adr) and docetaxel (Doc) - resistant MCF-7 cells (MCF-7/Doc), PTEN expression was significantly increased, whereas, Pgp expression was decreased after treatment with elemene (Zhang et al., 2014).

\section{SYNERGISTIC ANTICANCER EFFECTS}

In lung cancer cell lines NSCLC H460 and A549, elemene enhances cisplatin activity by increasing checkpoint kinase (CHK2) and reducing CDC2 (Li et al., 2013d). Elemene along with taxanes exhibited moderate synergistic antitumor activity against ovarian (A2780/CP70) and prostate (PC-3) carcinomas in vitro by increasing permeabilization of taxanes, micronuclear formation, and binding with efflux pumps along with upregulated expression of caspase-9 and p53 (Zou et al., 2013). It sensitized ovarian carcinoma cells to cisplatin treatment by down-regulating excision repair cross-complementation group-1 (ERCC-1) and XIAP through JNK pathway (Li et al., 2013a). It potentiated the activity of endostar, a recombinant human endostatin by inhibiting VEGF and MMP-2 leading to a decrease in malignant ascites formation in H22 murine model (Jiang et al., 2012).

\section{CONTRADICTORY EFFECTS OF ELEMENE}

In human gastric cancer cell lines MGC803 and SGC7901, protective autophagy was observed. According to the authors, the combination of autophagy inhibitors such as 3-methyladenine (3-MA) or chlorochine with elemene can induce apoptosis in the cancer cells by causing an increase in antitumor effects of elemene. Contraindicative to anticancer effects of elemene, when treated alone, it induced protective effects on gastric cancer cells (Liu et al., 2011). Similar observations of protective autophagy were attained against the breast cancer Bcap37 and MBA-MD231 cells (Guan et al., 2014).

\section{PROTECTIVE EFFECT AGAINST NORMAL CELLS}

Elemene exhibited low toxicity toward lung fibroblast (CCD-19Lu) and human bronchial epithelial (NL20) normal cell lines (Wang et al., 2005). In addition, elemene showed less toxicity to ovarian epithelial cell line (IOSE-397) (Li et al., 2005). It showed limited toxicity to macrophages and 
reduced infiltration of the same in rabbit in vivo model (Zhong et al., 2015).

\section{FUTURE DIRECTIONS}

Mechanistic approaches for the treatment of cancer, the modern epidemic, using elemene had made considerable progress over the recent past, resulting in decreased mortality rate. Simultaneously, the disease susceptibility and drug responses for elemene can be identified using specific molecular approaches. The studies of this kind are still at their infancy. Although available reports have cited elemene to be minimally toxic, this effect can be elucidated better. Further, elemene-induced liver injury has to be monitored. Adding to this, nano-formulations of elemene can be derived to enhance the bioavailability and efficacy of elemene, such that of nano-curcumin. This will eventually lead to a better, more specific and efficient anticancer therapy, devoid of side effects. Future studies that can elicit the exact role of elemene in anticancer therapy and novel, specific drug development methods are the need of the hour.

\section{CONCLUSION}

Elemene has been proven to possess anticancer activity. Decrease in mitochondrial potential, upregulation of pro-apoptotic signals

\section{REFERENCES}

Cai, D. Y., Gao, X., Wu, X. H., and Hong, T. T. (2013). Synergistic effect of beta-elemene injection combined paclitaxel injection on human breast cancer MB-468 cells: an in vitro study. Zhongguo Zhong Xi Yi Jie He Za Zhi 33, 978-982.

Chen, H., Shi, L., Cheng, Z. Y., Yao, L., Yang, Y. Y., and Pan, L. (2010). Effects of beta-elemene on proliferation and apoptosis of human multiple myeloma cell RPMI-8226. Zhongguo Shi Yan Xue Ye Xue Za Zhi 18, $368-371$.

Chen, W., Lu, Y., Wu, J., Gao, M., Wang, A., and Xu, B. (2011). Beta-elemene inhibits melanoma growth and metastasis via suppressing vascular endothelial growth factor-mediated angiogenesis. Cancer Chemother. Pharmacol. 67, 799-808. doi: 10.1007/s00280-010-1378-x

Chen, X., Wang, Y., Luo, H., Luo, Z., Zhang, T., Yang, N., et al. (2012). beta-elemene acts as an antitumor factor and downregulates the expression of survivin, $\mathrm{Bcl}-\mathrm{xL}$ and Mta-1. Mol. Med. Rep. 6, 989-995. doi: 10.3892/mmr.2012.1018

Dai, Z.-J., Tang, W., Lu, W.-F., Gao, J., Kang, H.-F., Ma, X.-B., et al. (2013). Antiproliferative and apoptotic effects of $\beta$-elemene on human hepatoma HepG2 cells. Cancer Cell Int. 13:27. doi: 10.1186/1475-2867-13-27

Dormond, O., Madsen, J. C., and Briscoe, D. M. (2007). The effects of mtor-Akt interactions on anti-apoptotic signaling in vascular endothelial cells. J. Biol. Chem. 282, 23679-23686. doi: 10.1074/jbc.M700563200

Frankfurt, O. S., and Krishan, A. (2003). Apoptosis-based drug screening and detection of selective toxicity to cancer cells. Anticancer Drugs 14, 555-561. doi: 10.1097/00001813-200308000-00008

Fu, T. H., Li, J. Y., Jing, Y. Y., Sun, P. J., and Bai, X. (2013). [Effect of elemene on reversing chemoresistance to adriamycin in human stomach cancer cell line]. Zhong Yao Cai 36, 601-603.

Guan, C., Liu, W., Yue, Y., Jin, H., Wang, X., and Wang, X.-J. (2014). Inhibitory effect of $\beta$-elemene on human breast cancer cells. Int. J. Clin. Exp. Pathol. 7, 3948-3956.

Guo, H.-Q., Zhang, G.-N., Wang, Y.-J., Zhang, Y.-K., Sodani, K., Talele, T. T., et al. (2014). $\beta$-Elemene, a compound derived from Rhizoma zedoariae, reverses multidrug resistance mediated by the ABCB1 transporter. Oncol. Rep. 31, 858-866. doi: 10.3892/or.2013.2870 and downregulation of anti-apoptotic signals are predominantly observed in cancer cells treated with elemene. Therefore, from the above results, it can be suggested that apoptosis has led to the anticancer activity of elemene (Table 1 and Figure 1). This is consistent with the molecular mechanism studied for other preclinical and clinical drugs. The mini-review based on the information presented, concludes that the $\beta$-elemene can be a precious candidate for future anticancer medications.

\section{AUTHOR CONTRIBUTIONS}

ZJ wrote the article; JJ edited the article; DL and PN revised the article; BC designed and approved the submission.

\section{ACKNOWLEDGMENTS}

This work was supported by the Key Medical Projects of Jiangsu Province (grant number BL2014078), Key Discipline of Jiangsu Province (2011-2015), Jiangsu Postdoctoral Science Research Fund (Grant no. 1601097B), National Natural Science Foundation of China (Grant No. NSFC 81503306) and Natural Science Foundation of Jiangsu Province (Grant No. BK20151045).

Hu, Z., Wu, H., Li, Y., Hou, Q., Wang, Y., Li, S., et al. (2015). $\beta$-Elemene inhibits the proliferation of esophageal squamous cell carcinoma by regulating long noncoding RNA-mediated inhibition of htert expression. Anticancer Drugs 26, 531-539. doi: 10.1097/CAD.0000000000000216

Jiang, S., Ling, C., Li, W., Jiang, H., Zhi, Q., and Jiang, M. (2016). Molecular mechanisms of anti-cancer activities of $\beta$-elemene: targeting hallmarks of cancer. Anti Cancer Agents Med. Chem. 16, 1426-1434. doi: 10.2174/ 1871520616666160211123424

Jiang, Z.-Y., Qin, S.-K., Yin, X.-J., Chen, Y.-L., and Zhu, L. (2012). Synergistic effects of Endostar combined with $\beta$-elemene on malignant ascites in a mouse model. Exp. Ther. Med. 4, 277-284.

Koul, H. K., Pal, M., and Koul, S. (2013). Role of p38 map kinase signal transduction in solid tumors. Genes Cancer 4, 342-359. doi: 10.1177/19476019135 07951

Lee, R. X., Li, Q. Q., and Reed, E. (2012). $\beta$-Elemene effectively suppresses the growth and survival of both platinum-sensitive and -resistant ovarian tumor cells. Anticancer Res. 32, 3103-3114.

Li, C. L., Chang, L., Guo, L., Zhao, D., Liu, H. B., Wang, Q. S., et al. (2014). betaelemene induces caspase-dependent apoptosis in human glioma cells in vitro through the upregulation of Bax and Fas/ FasL and downregulation of Bcl-2. Asian Pac. J. Cancer Prev. 15, 10407-10412. doi: 10.7314/APJCP.2014.15.23. 10407

Li, Q. Q., Lee, R. X., Liang, H., Wang, G., Li, J. M., Zhong, Y., et al. (2013a). $\beta$-Elemene enhances susceptibility to cisplatin in resistant ovarian carcinoma cells via downregulation of ERCC-1 and XIAP and inactivation of JNK. Int. J. Oncol. 43, 721-728. doi: 10.3892/ijo.2013.1996

Li, Q. Q., Lee, R. X., Liang, H., and Zhong, Y. (2013b). Anticancer activity of beta-elemene and its synthetic analogs in human malignant brain tumor cells. Anticancer Res. 33, 65-76.

Li, Q. Q., Lee, R. X., Liang, H., Zhong, Y., and Reed, E. (2013c). Enhancement of cisplatin-induced apoptosis by $\beta$-elemene in resistant human ovarian cancer cells. Med. Oncol. 30:424. doi: 10.1007/s12032-0120424-4

Li, Q. Q., Wang, G., Huang, F., Li, J. M., Cuff, C. F., and Reed, E. (2013d). Sensitization of lung cancer cells to cisplatin by beta-elemene is mediated 
through blockade of cell cycle progression: antitumor efficacies of beta-elemene and its synthetic analogs. Med. Oncol. 30:488. doi: 10.1007/s12032-013-0488-9

Li, Q. Q., Wang, G., Liang, H., Li, J. M., Huang, F., Agarwal, P. K., et al. (2013e). $\beta$-Elemene promotes cisplatin-induced cell death in human bladder cancer and other carcinomas. Anticancer Res. 33, 1421-1428.

Li, Q. Q., Wang, G., Huang, F., Banda, M., and Reed, E. (2010a). Antineoplastic effect of beta-elemene on prostate cancer cells and other types of solid tumour cells. J. Pharm. Pharmacol. 62, 1018-1027. doi: 10.1111/j.2042-7158.2010. 01135.x

Li, Q. Q., Wang, G., Reed, E., Huang, L., and Cuff, C. F. (2010b). Evaluation of cisplatin in combination with $\beta$-elemene as a regimen for prostate cancer chemotherapy. Basic Clin. Pharmacol. Toxicol. 107, 868-876. doi: 10.1111/j. 1742-7843.2010.00592.x

Li, Q. Q., Wang, G., Zhang, M., Cuff, C. F., Huang, L., and Reed, E. (2009). beta-Elemene, a novel plant-derived antineoplastic agent, increases cisplatin chemosensitivity of lung tumor cells by triggering apoptosis. Oncol. Rep. 22, 161-170. doi: 10.3892/or_00000420

Li, X., Lin, Z., Zhang, B., Guo, L., Liu, S., Li, H., et al. (2016). $\beta$-elemene sensitizes hepatocellular carcinoma cells to oxaliplatin by preventing oxaliplatin-induced degradation of copper transporter 1. Sci. Rep. 6:21010. doi: 10.1038/srep 21010

Li, X., Wang, G., Zhao, J., Ding, H., Cunningham, C., Chen, F., et al. (2005). Antiproliferative effect of beta-elemene in chemoresistant ovarian carcinoma cells is mediated through arrest of the cell cycle at the G2-M phase. Cell Mol. Life Sci. 62, 894-904. doi: 10.1007/s00018-005-5027-1

Liu, J., Zhang, Y., Qu, J., Xu, L., Hou, K., Zhang, J., et al. (2011). $\beta$-Elemene-induced autophagy protects human gastric cancer cells from undergoing apoptosis. BMC Cancer 11:183. doi: 10.1186/1471-240711-183

Saraste, A., and Pulkki, K. (2000). Morphologic and biochemical hallmarks of apoptosis. Cardiovasc. Res. 45, 528-537. doi: 10.1016/S0008-6363(99)00384-3

Siegel, R. L., Miller, K. D., and Jemal, A. (2016). Cancer statistics, 2016. CA Cancer J. Clin. 66, 7-30. doi: 10.3322/caac.21332

Son, J. K., Varadarajan, S., and Bratton, S. B. (2010). Trail-activated stress kinases suppress apoptosis through transcriptional upregulation of MCL-1. Cell Death Differ. 17, 1288-1301. doi: 10.1038/cdd.2010.9

Tao, L., Zhou, L., Zheng, L., and Yao, M. (2006). Elemene displays anti-cancer ability on laryngeal cancer cells in vitro and in vivo. Cancer Chemother. Pharmacol. 58, 24-34. doi: 10.1007/s00280-005-0137-x

Wang, G., Li, X., Huang, F., Zhao, J., Ding, H., Cunningham, C., et al. (2005). Antitumor effect of beta-elemene in non-small-cell lung cancer cells is mediated via induction of cell cycle arrest and apoptotic cell death. Cell Mol. Life Sci. 62, 881-893. doi: 10.1007/s00018-005-5017-3

Wu, X. S., Xie, T., Lin, J., Fan, H. Z., Huang-Fu, H. J., Ni, L. F., et al. (2009). An investigation of the ability of elemene to pass through the blood-brain barrier and its effect on brain carcinomas. J. Pharm. Pharmacol. 61, 1653-1656. doi: $10.1211 / \mathrm{jpp} / 61.12 .0010$

Xia, M., Knezevic, D., and Vassilev, L. T. (2011). p21 does not protect cancer cells from apoptosis induced by nongenotoxic p53 activation. Oncogene 30, 346-355. doi: 10.1038/onc. 2010.413

Yao, Y. Q., Ding, X., Jia, Y. C., Huang, C. X., Wang, Y. Z., and Xu, Y. H. (2008a). Anti-tumor effect of beta-elemene in glioblastoma cells depends on p38 MAPK activation. Cancer Lett. 264, 127-134. doi: 10.1016/j.canlet.2008.01.049

Yao, Y. Q., Xu, Y. H., Lu, J., Zhou, H. Y., and Wang, Y. Z. (2008b). [Effect of p38 MAPK on elemene-induced cell cycle arrest in C6 glioblastoma cells]. Zhonghua Yi Xue Za Zhi 88, 56-58.

Yu, Z. (2010). [Mechanism study of beta-elemene derivative (ET) in NB4 cell on apoptosis induction]. Zhongguo Zhong Yao Za Zhi 35, 1324-1327.

Yu, Z., Wang, R., Xu, L., Xie, S., Dong, J., and Jing, Y. (2011). ß-Elemene piperazine derivatives induce apoptosis in human leukemia cells through downregulation of c-FLIP and generation of ros. PLoS ONE 6:e15843. doi: 10.1371/journal.pone. 0015843

Zhang, F., Xu, L., Qu, X., Zhao, M., Jin, B., Kang, J., et al. (2011). Synergistic antitumor effect of beta-elemene and etoposide is mediated via induction of cell apoptosis and cell cycle arrest in non-small cell lung carcinoma cells. Mol. Med. Rep. 4, 1189-1193. doi: 10.3892/mmr.2011.537

Zhang, H., Xu, F., Xie, T., Jin, H., and Shi, L. (2012). beta-elemene induces glioma cell apoptosis by downregulating survivin and its interaction with hepatitis B X-interacting protein. Oncol. Rep. 28, 2083-2090. doi: 10.3892/or.2012. 2022

Zhang, J., Zhang, H., Chen, L., Sun, D. W., Mao, C., Chen, W., et al. (2014). $\beta$-Elemene reverses chemoresistance of breast cancer via regulating MDRrelated microrna expression. Cell Physiol. Biochem. 34, 2027-2037. doi: 10.1159/ 000366398

Zhang, Y., Mu, X.-D., Li, E.-Z., Luo, Y., Song, N., Qu, X.-J., et al. (2013). The role of $\mathrm{E} 3$ ubiquitin ligase $\mathrm{Cb}$ proteins in $\beta$-elemene reversing multi-drug resistance of human gastric adenocarcinoma cells. Int. J. Mol. Sci. 14, 10075-10089. doi: 10.3390/ijms 140510075

Zhao, J., Li, Q. Q., Zou, B., Wang, G., Li, X., Kim, J. E., et al. (2007). In vitro combination characterization of the new anticancer plant drug beta-elemene with taxanes against human lung carcinoma. Int. J. Oncol. 31, 241-252.

Zhao, S., Wu, J., Zheng, F., Tang, Q., Yang, L., Li, L., et al. (2015). $\beta$-elemene inhibited expression of Dna methyltransferase 1 through activation of ERK1/2 and Ampk $\alpha$ signalling pathways in human lung cancer cells: the role of Sp1. J. Cell Mol. Med. 19, 630-641. doi: 10.1111/jcmm.12476

Zhao, Y. F., Wang, C. R., Wu, Y. M., Ma, S. L., Ji, Y., and Lu, Y. J. (2011). P21 (waf1/cip1) is required for non-small cell lung cancer sensitive to Gefitinib treatment. Biomed. Pharmacother. 65, 151-156. doi: 10.1016/j.biopha.2011. 02.009

Zheng, C.-P., Tong, X.-M., Yao, H.-P., Yang, J., Xu, J., Cai, X.-P., et al. (2009). [betaelemene enhances aclarubicin-induced apoptotic effect in Hl-60 cells and its mechanism.]. Zhonghua Xue Ye Xue Za Zhi 30, 821-824.

Zhong, Y., Liu, J., Huo, W. M., Duan, W. L., Wang, X., and Shang, J. (2015). betaElemene reduces the progression of atherosclerosis in rabbits. Chin. J. Nat. Med. 13, 415-420. doi: 10.1016/S1875-5364(15)30034-0

Zhou, H. Y., Shen, J. K., Hou, J. S., Qiu, Y. M., and Luo, Q. Z. (2003). [Experimental study on apoptosis induced by elemene in glioma cells]. Ai Zheng 22, 959-963.

Zhou, J., He, L.-L., Ding, X.-F., Yuan, Q.-Q., Zhang, J.-X., Liu, S.-C., et al. (2016). Combinatorial antitumor effect of rapamycin and $\beta$-elemene in follicular thyroid cancer cells. BioMed. Res. Int. 2016:6723807. doi: 10.1155/2016/6723807

Zhu, T., Xu, Y., Dong, B., Zhang, J., Wei, Z., and Yao, Y. (2011a). beta-elemene inhibits proliferation of human glioblastoma cells through the activation of glia maturation factor beta and induces sensitization to cisplatin. Oncol. Rep. 26, 405-413. doi: 10.3892/or.2011.1276

Zhu, T., Zhao, Y., Zhang, J., Li, L., Zou, L., Yao, Y., et al. (2011b). $\beta$-Elemene inhibits proliferation of human glioblastoma cells and causes cell-cycle G0/G1 arrest via mutually compensatory activation of MKK3 and MKK6. Int. J. Oncol. 38, 419-426. doi: 10.3892/ijo.2010.855

Zhu, T. Z., Li, X. M., Luo, L. H., Xu, Y. H., Cao, P., Liu, Y., et al. (2014). betaElemene inhibits proliferation through crosstalk between glia maturation factor beta and extracellular signalregulated kinase $1 / 2$ and impairs drug resistance to temozolomide in glioblastoma cells. Mol. Med. Rep. 10, 1122-1128. doi: 10.3892/mmr.2014.2273

Zou, B., Li, Q. Q., Zhao, J., Li, J. M., Cuff, C. F., and Reed, E. (2013). beta-Elemene and taxanes synergistically induce cytotoxicity and inhibit proliferation in ovarian cancer and other tumor cells. Anticancer Res. 33, 929-940.

Conflict of Interest Statement: The authors declare that the research was conducted in the absence of any commercial or financial relationships that could be construed as a potential conflict of interest.

Copyright (®) 2017 Jiang, Jacob, Loganathachetti, Nainangu and Chen. This is an open-access article distributed under the terms of the Creative Commons Attribution License (CC BY). The use, distribution or reproduction in other forums is permitted, provided the original author(s) or licensor are credited and that the original publication in this journal is cited, in accordance with accepted academic practice. No use, distribution or reproduction is permitted which does not comply with these terms. 